\title{
Evaluation of Bone Thickness Changes Associated with En-Masse Retraction Facilitated By Corticotomy and Piezocision Using Cone Beam Computed Tomography.
}

\author{
Rehab A Khalil ${ }^{\star}$, Waleed M Abbas ${ }^{\star *}$, Waleed El-Sayed Refaat ${ }^{\star \star \star}$, \\ Ahmed A Ramadan ${ }^{* \star *}$
}

\section{ABSTRACT:}

Objective: The purpose of this study was to assess the bone thickness changes accompanied en-masse retraction assisted by corticotomy and piezocision by using $C B C T$ (cone beam computed tomography). Subjects and Methods: Twenty subjects having Class I malocclusion with bimaxillary protrusion were included in the study. The patients were randomly divided into two equal groups; group I: flap group and group II: piezocision group. CBCT images were obtained before treatment (T1) and after space closure (T2). The alveolar bone thickness was assessed on the labial and lingual sides at the cervical, middle and apical thirds of the roots of the upper and lower anteriors. Results: Group I demonstrated a significant increase in the cervical labial bone thickness of the upper incisors while no significant change was detected in group II. Both groups revealed a significant decrease in the 6one thickness of the cervical labial third of the lower anteriors. The labial bone thickness of the middle third showed no significant change except the upper left lateral incisor which revealed a significant decrease in group I. The labial bone thickness at the apical third showed no significant change in 6oth groups. For both groups, the lingual bone thickness showed a significant decrease for all anteriors in the cervical and middle thirds. The apical third revealed no significant changes except the canines which showed a significant decrease in group I. Conclusion: En-masse retraction aided with either corticotomy or piezocision in bimaxillary protrusion cases showed significant bone thickness changes on the labial and lingual sides.

\section{INTRODUCTION}

Bimaxillary protrusion is a widespread malocclusion that interferes with facial esthetic due to protrusion of anterior teeth and lips, lip incompetence and convex profile. ${ }^{(1)}$ The goal of treatment is to retract the anterior teeth to improve facial esthetics and provide functional balance. This could be reached through anterior segmental osteotomy or by orthodontic treatment which involved extraction of four first premolars and retraction of anterior teeth with maximum anchorage. This treatment modality may not be the proper technique for

\footnotetext{
* Assistant Lecturer, Department of Orthodontics, Faculty of Dentistry, Beni-Suef University.

** Lecturer of Oral Medicine and periodontology, Faculty of Dentistry, Ain Shams University.

*** Professor of Orthodontics, Faculty of Dentistry, Suez Canal University.
} 
patients with bimaxillary protrusion ${ }^{(2)}$ as excessive retraction of anterior teeth could be associated with root resorption and alveolar bone loss due to the presence of thin elongated anterior alveoli and bony defects before treatment. $^{(3)}$

Lateral cephalograms were used to determine the alveolar bone changes associated with tooth movement. ${ }^{(4,5)}$ With the advent of CT (computed tomography) and CBCT (Cone beam computed tomography), craniofacial structures could be evaluated and visualized in three dimensions. A CT scan evaluating the changes in the labial and lingual alveolar bone thickness associated with retraction of anterior teeth in bimaxillary protrusion cases revealed that adverse sequelae could be observed when forcing the teeth against the cortical plate and so very light forces and long-term activations may be required to allow alveolar bone adaptation. ${ }^{(6)}$

En-masse retraction of anterior teeth in bimaxillary protrusion cases showed a decrease of alveolar bone area, vertical bone level on the palatal side, root length and root area of maxillary central and lateral incisors as detected by CBCT. ${ }^{(7)}$ Other studies ${ }^{(8,9)}$ reported significant increase of the labial bone thickness at the crestal level as the incisors were retracted. A decrease in palatal bone thickness after incisor retraction was documented in previous studies. ${ }^{(10-12)} \mathrm{A}$ dehiscence on the palatal alveolar bone of the maxillary incisors after retraction was corrected after a retention period of approximately 10 years. ${ }^{(12)}$

Previous studies ${ }^{(3,7,13)}$ recommended retraction of anterior teeth combined with alveolar corticotomy to minimize the associated risks accompanied retraction of anterior teeth. Few articles had documented corticotomy-assisted orthodontic correction of bimaxillary protrusion and no data regarding the effect of piezocision on en- masse retraction of the lower arch was found in the available literature. Therefore, the aim of this study was to evaluate the bone thickness changes associated with en-masse retraction facilitated by corticotomy and piezocision by using CBCT.

\section{SUBJECTS AND METHODS}

The sample of this study included twenty subjects (19 females, 1 male) selected from the out patients of Orthodontic clinic, Faculty of Dentistry, Suez Canal University. All the patients were having Class I malocclusion with bimaxillary protrusion ranged from 15 - 25 years old. The patients exhibited fair oral hygiene with no systemic diseases.

The sample was randomly (simple randomization through computer generated schedule in Excel with 1:1 allocation ratio) divided into two equal groups:

Group I: flap group.

Group II: piezocision group (flapless corticotomy).

Allocation concealment was designed and blinding was restricted to the outcome assessment. A consent form was signed after informing the subjects and parents about the surgical procedures. The following records were obtained before and after treatment: extraoral and intraoral photographs, orthodontic study models, panoramic radiographs and lateral cephalometric radiographs.

CBCT images were obtained before treatment (T1) and after space closure (T2) by using Scanora 3Dx machine.*

The upper and lower arches were bonded with 0.022 inch MBT metal bracket.**

\footnotetext{
*Soredex, Tuusula, Finland.

* *Gemini MBT metal bracket, 3M Unitek Orthodontic Products, Monrovia, CA.
} 
Anchorage reinforcement was achieved by the use of Vector TAS miniscrews* $(1.4 \mathrm{~mm}$ in diameter and $8 \mathrm{~mm}$ in length) that were inserted between the first molars and second premolars. Crimpable** hooks $(10 \mathrm{~mm}$ in length) were attached to the archwires between the lateral incisors and canines.

\section{Surgical procedures:}

Surgery was performed in the upper and lower arches under the use of local anaesthesia. The four first premolars were extracted just before surgery.

Group I: flap group.

A full-thickness mucoperiosteal flap was reflected by the use of mucoperiosteal elevator beyond the apices of the upper and lower six anterior teeth after cutting a submarginal scalloped flap design. A piezosurgery knife*** was used to cut interproximal vertical grooves (Figure 1) with a depth of $3 \mathrm{~mm}$ (verified by the millimetric signs of the piezosurgery knife). The flap was repositioned and sutured with the interrupted technique.

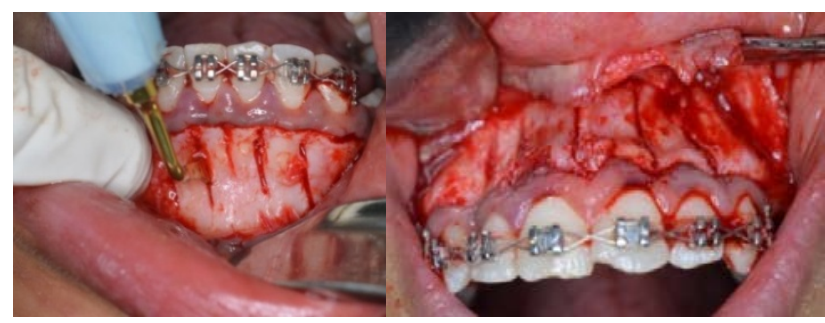

Figure (1): Interproximal vertical corticotomy cuts.

Group II: piezocision group (flapless corticotomy).

Cortical alveolar interproximal incisions (3mm in depth) were cut with the same piezosurgery knife through vertical interproximal gingival incisions that were cut $4 \mathrm{~mm}$ apical to the interdental papillae to $2-3 \mathrm{~mm}$ apical to the mucogingival junction of the upper and lower six anterior teeth. The incisions were sutured with the interrupted technique.

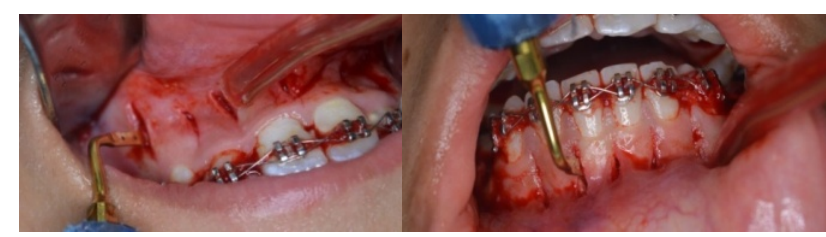

Figure (2): Piezocision cuts.

En-masse retraction was initiated immediately after the surgical procedures on $0.017 \times 0.025$ ', stainless steel wires by the use of $9 \mathrm{~mm} \mathrm{NiTi****}$ closed coil springs that were inserted from the miniscrews to the crimpable hooks.

\section{CBCT analysis:}

CBCT images were obtained before treatment (T1) and after space closure (T2) to evaluate the changes in the alveolar bone thickness on the labial and lingual sides of the upper and lower six anteriors after retraction.

The files were acquired in Digital Imaging and Communications in Medicine (DICOM) format and imported into InVivo-Dental software (Version 5.3, Anatomage, San Jose, Calif). On the MPR (Multiplanar reformatted) screen, in order to standardize the measurements, the sagittal reference plane was adjusted on the axial slice to be passing through the middle of the tooth labiolingually. On the sagittal slice, the coronal reference plane was adjusted to be passing through the root apex and the incisal edge (Figure 3). Measurements were performed on the sagittal slice. The thickness of the labial and lingual alveolar plates was measured for each tooth as described by Sarikaya et al., ${ }^{(6)}$ at the site adjacent to the widest point of the labiolingual root (Figure 4) in three slices separated

*Ormco, Glendora, CA.

**Hubit, Gyeonggi-do, Korea.

*** Variosurg, NSK, Tokyo, Japan.

****Modern Orthodontics, Ludhiana, India. 
by $3 \mathrm{~mm}$ (S1, S2 and S3) and started 3mm apical to the CEJ (cementoenamel junction).

The alveolar bone thickness of the labial (La) and lingual ( $\mathrm{Li}$ ) plates was assessed at the cervical level (S1; La1, Li1), midroot level (S2; La2, Li2) and apical level (S3; La3, Li3). All measurements were taken by one investigator who was blinded to the study groups.

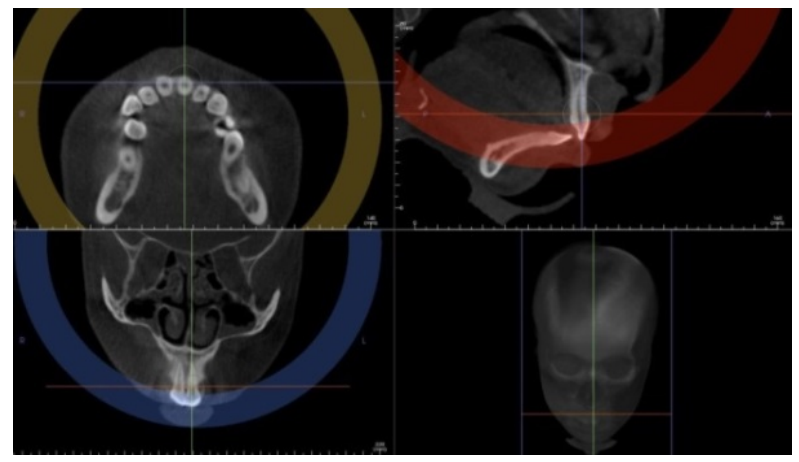

Figure (3): Standardization.

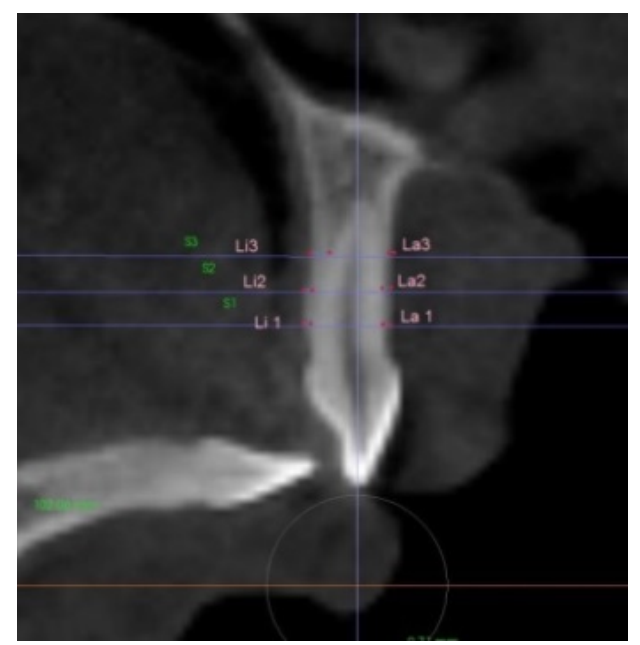

Figure (4): Measurements of bone thickness at the cervical (S1), middle (S2) and apical (S3) thirds.

\section{RESULTS}

Paired t-test was used to compare the bone thickness changes between $\mathrm{T} 1$ (pretreatment) and T2 (space closure) in each group. The teeth were numbered according to the Federation Dentaire International Numbering System (FDI).

\section{1-Evaluation of the labial bone thickness} changes:

\section{Group I:}

For the cervical third, there was a significant increase of the labial bone thickness of the upper incisors, while the upper canines showed no significant change. The lower anteriors showed a significant decrease of the labial bone thickness at the cervical third. The labial bone thickness of the middle third showed no significant change except the upper left lateral incisor which revealed a significant decrease. The labial bone thickness at the apical third showed no significant change (Table 1).

\section{Group II:}

For the cervical third, the upper anteriors showed no significant change in the labial bone thickness, while the lower anteriors revealed a significant decrease. The bone thickness in the middle and apical thirds demonstrated no significant change (Table 2).

\section{2- Evaluation of the lingual bone thickness changes:}

\section{Group I:}

There was a significant decrease in the lingual bone thickness for all anteriors in the cervical and middle thirds. The bone thickness in the apical third showed no significant change for the upper and lower incisors, while the canines showed a significant decrease (Table 3).

\section{Group II:}

There was a significant decrease in the lingual bone thickness for all anteriors in the cervical and middle thirds. The bone thickness in the apical third showed no significant change (Table 4). 
Table (1): Mean, standard deviation (SD) of the pre and post values, paired differences and results of the Paired t-test of the labial bone thickness (in $\mathrm{mm}$ ) for group I.

\begin{tabular}{|c|c|c|c|c|c|c|c|c|c|}
\hline \multicolumn{3}{|c|}{ labial } & \multicolumn{2}{|c|}{ pre } & \multicolumn{2}{|c|}{ post } & \multicolumn{2}{|c|}{$\begin{array}{l}\text { Paired Differences [Labial } \\
\text { (Post) -Labial (Pre)] }\end{array}$} & \multirow[t]{2}{*}{ p-value } \\
\hline & & & Mean & SD & Mean & SD & Mean & SD & \\
\hline \multirow{36}{*}{$\begin{array}{c}\text { Group I } \\
\text { (Flap) }\end{array}$} & \multirow[t]{12}{*}{ Cervical } & 11 & 1.51 & 0.58 & 2.17 & 0.79 & 0.67 & 0.76 & $0.022 *$ \\
\hline & & 12 & 1.03 & 0.34 & 1.71 & 1.00 & 0.68 & 0.94 & $0.049 *$ \\
\hline & & 13 & 1.50 & 1.04 & 1.07 & 0.31 & -0.44 & 0.96 & $0.184 \mathrm{NS}$ \\
\hline & & 21 & 1.41 & 0.32 & 2.16 & 0.99 & 0.75 & 0.94 & 0.033* \\
\hline & & 22 & 1.14 & 0.31 & 1.71 & 0.72 & 0.58 & 0.77 & $0.042^{*}$ \\
\hline & & 23 & 1.29 & 0.75 & 1.38 & 0.87 & 0.09 & 0.66 & $0.675 \mathrm{NS}$ \\
\hline & & 31 & 1.11 & 0.36 & 0.72 & 0.24 & -0.39 & 0.44 & $0.02 *$ \\
\hline & & 32 & 1.05 & 0.34 & 0.71 & 0.28 & -0.35 & 0.36 & $0.013^{*}$ \\
\hline & & 33 & 0.97 & 0.34 & 0.69 & 0.24 & -0.27 & 0.37 & $0.046^{*}$ \\
\hline & & 41 & 0.96 & 0.48 & 0.61 & 0.16 & -0.35 & 0.48 & $0.05 *$ \\
\hline & & 42 & 1.10 & 0.32 & 0.67 & 0.23 & -0.43 & 0.42 & $0.01 *$ \\
\hline & & 43 & 0.94 & 0.13 & 0.66 & 0.34 & -0.29 & 0.39 & $0.048^{*}$ \\
\hline & \multirow[t]{12}{*}{ Middle } & 11 & 1.58 & 0.82 & 1.83 & 0.99 & 0.25 & 0.66 & $0.263 \mathrm{NS}$ \\
\hline & & 12 & 1.30 & 0.34 & 1.52 & 0.74 & 0.22 & 0.75 & $0.376 \mathrm{NS}$ \\
\hline & & 13 & 1.48 & 1.05 & 1.11 & 0.42 & -0.37 & 1.14 & $0.327 \mathrm{NS}$ \\
\hline & & 21 & 1.62 & 0.56 & 1.52 & 0.71 & -0.10 & 0.41 & $0.470 \mathrm{NS}$ \\
\hline & & 22 & 1.49 & 0.42 & 1.12 & 0.34 & -0.36 & 0.45 & $0.032 *$ \\
\hline & & 23 & 1.39 & 0.54 & 1.25 & 0.36 & -0.14 & 0.26 & $0.126 \mathrm{NS}$ \\
\hline & & 31 & 0.97 & 0.25 & 0.86 & 0.38 & -0.11 & 0.32 & $0.298 \mathrm{NS}$ \\
\hline & & 32 & 0.88 & 0.20 & 1.10 & 0.48 & 0.22 & 0.46 & $0.169 \mathrm{NS}$ \\
\hline & & 33 & 0.88 & 0.26 & 0.96 & 0.48 & 0.07 & 0.48 & $0.641 \mathrm{NS}$ \\
\hline & & 41 & 0.89 & 0.31 & 0.96 & 0.30 & 0.08 & 0.51 & $0.649 \mathrm{NS}$ \\
\hline & & 42 & 1.00 & 0.27 & 1.04 & 0.45 & 0.04 & 0.48 & $0.779 \mathrm{NS}$ \\
\hline & & 43 & 0.83 & 0.24 & 0.94 & 0.51 & 0.11 & 0.48 & $0.478 \mathrm{NS}$ \\
\hline & \multirow[t]{12}{*}{ Apical } & 11 & 1.51 & 0.56 & 1.65 & 0.97 & 0.15 & 0.95 & $0.639 \mathrm{NS}$ \\
\hline & & 12 & 1.16 & 0.41 & 1.32 & 0.61 & 0.16 & 0.59 & $0.421 \mathrm{NS}$ \\
\hline & & 13 & 1.50 & 1.16 & 1.18 & 0.36 & -0.32 & 1.31 & 0.459 NS \\
\hline & & 21 & 1.45 & 0.31 & 1.33 & 0.68 & -0.12 & 0.63 & $0.569 \mathrm{NS}$ \\
\hline & & 22 & 1.20 & 0.28 & 1.09 & 0.25 & -0.11 & 0.22 & $0.152 \mathrm{NS}$ \\
\hline & & 23 & 1.24 & 0.24 & 1.08 & 0.32 & -0.16 & 0.32 & $0.161 \mathrm{NS}$ \\
\hline & & 31 & 1.28 & 0.36 & 1.50 & 0.89 & 0.22 & 0.66 & $0.328 \mathrm{NS}$ \\
\hline & & 32 & 1.09 & 0.46 & 1.27 & 0.47 & 0.18 & 0.36 & $0.148 \mathrm{NS}$ \\
\hline & & 33 & 1.21 & 0.51 & 1.31 & 0.69 & 0.10 & 0.66 & $0.642 \mathrm{NS}$ \\
\hline & & 41 & 1.12 & 0.37 & 1.59 & 0.95 & 0.47 & 0.99 & $0.172 \mathrm{NS}$ \\
\hline & & 42 & 0.94 & 0.38 & 1.28 & 0.57 & 0.35 & 0.71 & $0.158 \mathrm{NS}$ \\
\hline & & 43 & 1.10 & 0.28 & 1.31 & 0.64 & 0.21 & 0.49 & $0.203 \mathrm{NS}$ \\
\hline
\end{tabular}

\footnotetext{
*= Significant, NS=Non-significant.
} 
Table (2): Mean, standard deviation (SD) of the pre and post values, paired differences and results of the Paired t-test of the labial bone thickness (in $\mathrm{mm}$ ) for group II.

\begin{tabular}{|c|c|c|c|c|c|c|c|c|c|}
\hline \multirow{2}{*}{\multicolumn{3}{|c|}{ labial }} & \multicolumn{2}{|c|}{ pre } & \multicolumn{2}{|c|}{ post } & \multicolumn{2}{|c|}{$\begin{array}{l}\text { Paired Differences [Labial } \\
\text { (Post) -Labial (Pre)] }\end{array}$} & \multirow[t]{2}{*}{ p-value } \\
\hline & & & Mean & SD & Mean & SD & Mean & SD & \\
\hline \multirow{36}{*}{$\begin{array}{c}\text { Group II } \\
\text { (Flapless) }\end{array}$} & \multirow{12}{*}{ Cervical } & 11 & 1.40 & 0.23 & 1.34 & 0.23 & -0.06 & 0.27 & $0.490 \mathrm{NS}$ \\
\hline & & 12 & 1.00 & 0.27 & 0.97 & 0.37 & -0.03 & 0.35 & $0.777 \mathrm{NS}$ \\
\hline & & 13 & 1.28 & 0.37 & 1.14 & 0.42 & -0.15 & 0.61 & $0.465 \mathrm{NS}$ \\
\hline & & 21 & 1.38 & 0.23 & 1.36 & 0.30 & -0.02 & 0.26 & $0.838 \mathrm{NS}$ \\
\hline & & 22 & 1.15 & 0.29 & 1.06 & 0.24 & -0.09 & 0.36 & $0.447 \mathrm{NS}$ \\
\hline & & 23 & 1.27 & 0.42 & 1.13 & 0.40 & -0.14 & 0.48 & $0.372 \mathrm{NS}$ \\
\hline & & 31 & 1.02 & 0.43 & 0.73 & 0.13 & -0.29 & 0.40 & $0.048^{*}$ \\
\hline & & 32 & 1.00 & 0.33 & 0.79 & 0.25 & -0.21 & 0.26 & $0.031 *$ \\
\hline & & 33 & 1.20 & 0.39 & 0.76 & 0.13 & -0.44 & 0.43 & $0.01 *$ \\
\hline & & 41 & 1.01 & 0.42 & 0.62 & 0.16 & -0.39 & 0.39 & $0.011 *$ \\
\hline & & 42 & 1.03 & 0.33 & 0.75 & 0.20 & -0.27 & 0.31 & $0.022 *$ \\
\hline & & 43 & 1.10 & 0.39 & 0.75 & 0.10 & -0.35 & 0.37 & $0.014 *$ \\
\hline & \multirow{12}{*}{ Middle } & 11 & 1.20 & 0.21 & 1.18 & 0.25 & -0.02 & 0.20 & $0.797 \mathrm{NS}$ \\
\hline & & 12 & 1.21 & 0.28 & 1.44 & 0.83 & 0.22 & 0.91 & $0.455 \mathrm{NS}$ \\
\hline & & 13 & 1.49 & 0.40 & 1.26 & 0.31 & -0.23 & 0.47 & $0.150 \mathrm{NS}$ \\
\hline & & 21 & 1.33 & 0.33 & 1.24 & 0.38 & -0.09 & 0.32 & $0.384 \mathrm{NS}$ \\
\hline & & 22 & 1.36 & 0.26 & 1.26 & 0.32 & -0.11 & 0.20 & $0.127 \mathrm{NS}$ \\
\hline & & 23 & 1.32 & 0.21 & 1.12 & 0.25 & -0.20 & 0.36 & $0.110 \mathrm{NS}$ \\
\hline & & 31 & 1.05 & 0.42 & 0.88 & 0.27 & -0.17 & 0.26 & $0.07 \mathrm{NS}$ \\
\hline & & 32 & 0.85 & 0.28 & 0.83 & 0.27 & -0.02 & 0.23 & $0.787 \mathrm{NS}$ \\
\hline & & 33 & 1.00 & 0.13 & 1.00 & 0.45 & 0.00 & 0.46 & $0.995 \mathrm{NS}$ \\
\hline & & 41 & 0.90 & 0.35 & 0.81 & 0.23 & -0.09 & 0.32 & $0.408 \mathrm{NS}$ \\
\hline & & 42 & 1.03 & 0.21 & 0.89 & 0.29 & -0.14 & 0.28 & $0.157 \mathrm{NS}$ \\
\hline & & 43 & 0.98 & 0.20 & 0.84 & 0.23 & $\begin{array}{l}-0.14 \\
\end{array}$ & 0.23 & $0.089 \mathrm{NS}$ \\
\hline & \multirow{12}{*}{ Apical } & 11 & 1.33 & 0.18 & 1.24 & 0.19 & -0.10 & 0.17 & $0.097 \mathrm{NS}$ \\
\hline & & 12 & 1.15 & 0.16 & 1.20 & 0.45 & 0.05 & 0.43 & $0.724 \mathrm{NS}$ \\
\hline & & 13 & 1.48 & 0.37 & 1.32 & 0.43 & -0.17 & 0.52 & $0.343 \mathrm{NS}$ \\
\hline & & 21 & 1.33 & 0.32 & 1.22 & 0.29 & $\begin{array}{l}-0.11 \\
\end{array}$ & 0.35 & $0.344 \mathrm{NS}$ \\
\hline & & 22 & 1.23 & 0.37 & 1.18 & 0.67 & -0.05 & 0.60 & $0.814 \mathrm{NS}$ \\
\hline & & 23 & 1.22 & 0.11 & 1.13 & 0.18 & -0.09 & 0.23 & $0.261 \mathrm{NS}$ \\
\hline & & 31 & 1.21 & 0.26 & 1.17 & 0.21 & -0.03 & 0.29 & $0.718 \mathrm{NS}$ \\
\hline & & 32 & 1.06 & 0.34 & 1.00 & 0.28 & -0.05 & 0.33 & $0.634 \mathrm{NS}$ \\
\hline & & 33 & 1.26 & 0.32 & 1.11 & 0.16 & -0.16 & 0.32 & $0.158 \mathrm{NS}$ \\
\hline & & 41 & 1.13 & 0.35 & 1.18 & 0.32 & 0.05 & 0.14 & $0.260 \mathrm{NS}$ \\
\hline & & 42 & 1.14 & 0.17 & 1.13 & 0.26 & -0.01 & 0.29 & $0.949 \mathrm{NS}$ \\
\hline & & 43 & 1.13 & 0.19 & 1.12 & 0.23 & $\begin{array}{l}-0.01 \\
\end{array}$ & 0.30 & $0.895 \mathrm{NS}$ \\
\hline
\end{tabular}

*= Significant, NS=Non-significant.

\section{DISCUSSION}

Bimaxillary dentoalveolar protrusion is a current orthodontic problem. Patients with bimaxillary protrusion usually seek orthodontic treatment for improving their facial esthetics. Corticotomy and piezocision were performed in the present study to facilitate en-masse retraction in twenty subjects (19 females, 1 male) having Class I bimaxillary protrusion with age ranged from 15-25 years old.

One of the goals of orthodontic treatment is to obtain the desired tooth movement while minimizing the undesirable consequences to the alveolar bone. The introduction of cone beam computed tomography (CBCT) into orthodontic practice provided the advantage of 
evaluating hard tissue changes in three dimensions. ${ }^{(3)}$ The results of the present study for group I agreed with previous studies ${ }^{(4,5,9,11)}$ which reported increased labial bone thickness at the cervical level of the root after incisor retraction. The current finding matched the clinical observation of Mimura $^{(2)}$ and
Bae et al., ${ }^{(12)}$ who reported the presence of a bony spicule in the maxillary labial alveolar bone during incisor retraction. These results contradicted the theory of De Angelis ${ }^{(14)}$ which suggested that the alveolar bone retained its structural characteristics and size as it moved.

Table (3): Mean, standard deviation (SD) of the pre and post values,paired differences and results of the Paired t-test of the lingual bone thickness (in $\mathrm{mm}$ ) for group I.

\begin{tabular}{|c|c|c|c|c|c|c|c|c|c|}
\hline \multicolumn{3}{|c|}{ lingual } & \multicolumn{2}{|c|}{ pre } & \multicolumn{2}{|c|}{ post } & \multicolumn{2}{|c|}{$\begin{array}{l}\text { Paired Differences [Lingual } \\
\text { (Post) -Lingual (Pre)] }\end{array}$} & \multirow[t]{2}{*}{ p-value } \\
\hline & & & Mean & SD & Mean & SD & Mean & SD & \\
\hline \multirow{12}{*}{\multicolumn{2}{|c|}{ Cervical }} & 11 & 1.50 & 0.41 & 0.90 & 0.54 & -0.60 & 0.57 & $0.008^{*}$ \\
\hline & & 12 & 1.19 & 0.35 & 0.80 & 0.27 & -0.40 & 0.44 & $0.019 *$ \\
\hline & & 13 & 1.33 & 1.07 & 0.63 & 0.23 & -0.70 & 0.97 & $0.049 *$ \\
\hline & & 21 & 1.45 & 0.35 & 0.71 & 0.50 & -0.74 & 0.53 & $0.002 *$ \\
\hline & & 22 & 1.26 & 0.34 & 0.82 & 0.43 & -0.45 & 0.62 & $0.049 *$ \\
\hline & & 23 & 1.28 & 0.36 & 0.79 & 0.45 & -0.49 & 0.52 & $0.015^{*}$ \\
\hline & & 31 & 1.15 & 0.32 & 0.60 & 0.14 & -0.55 & 0.35 & $0.001 *$ \\
\hline & & 32 & 1.28 & 0.26 & 0.74 & 0.25 & -0.55 & 0.31 & $\leq 0.001^{*}$ \\
\hline & & 33 & 1.36 & 0.36 & 0.89 & 0.42 & -0.48 & 0.61 & $0.036^{*}$ \\
\hline & & 41 & 1.00 & 0.30 & 0.66 & 0.08 & -0.34 & 0.31 & $0.008 *$ \\
\hline & & 42 & 1.29 & 0.27 & 0.73 & 0.24 & -0.55 & 0.38 & $0.001^{*}$ \\
\hline & & 43 & 1.39 & 0.33 & 1.00 & 0.41 & -0.39 & 0.52 & $0.04 *$ \\
\hline \multirow{24}{*}{$\begin{array}{l}\text { Group I } \\
\text { (Flap) }\end{array}$} & \multirow[t]{12}{*}{ Middle } & 11 & 2.28 & 0.38 & 1.78 & 0.81 & -0.51 & 0.63 & $0.031 *$ \\
\hline & & 12 & 1.71 & 0.28 & 1.32 & 0.23 & -0.39 & 0.34 & $0.006^{*}$ \\
\hline & & 13 & 2.43 & 1.24 & 1.81 & 0.57 & -0.62 & 0.84 & $0.045 *$ \\
\hline & & 21 & 2.17 & 0.59 & 1.76 & 0.71 & -0.41 & 0.53 & $0.035 *$ \\
\hline & & 22 & 1.94 & 0.43 & 1.52 & 0.52 & -0.42 & 0.52 & $0.032 *$ \\
\hline & & 23 & 2.40 & 0.56 & 1.81 & 0.42 & -0.59 & 0.72 & $0.029 *$ \\
\hline & & 31 & 1.10 & 0.27 & 0.85 & 0.20 & -0.25 & 0.28 & $0.02 *$ \\
\hline & & 32 & 1.31 & 0.50 & 1.02 & 0.39 & -0.29 & 0.30 & $0.016^{*}$ \\
\hline & & 33 & 1.58 & 0.53 & 1.20 & 0.51 & -0.38 & 0.35 & $0.007 *$ \\
\hline & & 41 & 1.18 & 0.26 & 0.95 & 0.34 & -0.24 & 0.33 & $0.048 *$ \\
\hline & & 42 & 1.33 & 0.51 & 0.98 & 0.31 & -0.35 & 0.37 & $0.016^{*}$ \\
\hline & & 43 & 1.62 & 0.41 & 1.19 & 0.46 & -0.43 & 0.26 & $0.001^{*}$ \\
\hline & \multirow[t]{12}{*}{ Apical } & 11 & 3.14 & 0.95 & 3.56 & 2.11 & 0.43 & 1.49 & $0.391 \mathrm{NS}$ \\
\hline & & 12 & 2.80 & 0.82 & 2.58 & 0.95 & -0.22 & 1.02 & $0.517 \mathrm{NS}$ \\
\hline & & 13 & 3.62 & 1.56 & 2.90 & 1.00 & -0.72 & 0.99 & $0.047 *$ \\
\hline & & 21 & 2.96 & 0.63 & 3.81 & 1.91 & 0.85 & 1.65 & $0.137 \mathrm{NS}$ \\
\hline & & 22 & 2.93 & 0.61 & 2.77 & 0.72 & -0.16 & 0.62 & $0.433 \mathrm{NS}$ \\
\hline & & 23 & 3.75 & 1.03 & 2.85 & 0.69 & -0.90 & 0.85 & $0.009 *$ \\
\hline & & 31 & 1.54 & 0.53 & 1.46 & 0.38 & -0.08 & 0.42 & $0.559 \mathrm{NS}$ \\
\hline & & 32 & 1.51 & 0.72 & 1.40 & 0.46 & -0.12 & 0.49 & $0.47 \quad \mathrm{NS}$ \\
\hline & & 33 & 1.75 & 0.70 & 1.37 & 0.57 & -0.38 & 0.47 & $0.031^{*}$ \\
\hline & & 41 & 1.49 & 0.62 & 1.32 & 0.56 & -0.17 & 0.54 & $0.339 \mathrm{NS}$ \\
\hline & & 42 & 1.69 & 0.89 & 1.51 & 0.60 & -0.19 & 0.45 & $0.229 \mathrm{NS}$ \\
\hline & & 43 & 1.87 & 0.99 & 1.68 & 0.87 & -0.19 & 0.21 & $0.02 *$ \\
\hline
\end{tabular}

*= Significant, NS=Non-significant. 
Table (4): Mean, standard deviation (SD) of the pre and post values, paired differences and results of the Paired t-test of the lingual bone thickness (in $\mathrm{mm}$ ) for group II.

\begin{tabular}{|c|c|c|c|c|c|c|c|c|c|}
\hline \multicolumn{3}{|c|}{ lingual } & \multicolumn{2}{|c|}{ pre } & \multicolumn{2}{|c|}{ post } & \multicolumn{2}{|c|}{$\begin{array}{l}\text { Paired Differences [Lingual } \\
\text { (Post) - Lingual (Pre)] }\end{array}$} & \multirow[t]{2}{*}{ p-value } \\
\hline & & & Mean & SD & Mean & SD & Mean & SD & \\
\hline \multirow{36}{*}{$\begin{array}{l}\text { Group II } \\
\text { (Flapless) }\end{array}$} & \multirow[t]{12}{*}{ Cervical } & 11 & 1.85 & 0.62 & 0.93 & 0.25 & -0.92 & 0.54 & $\leq 0.001^{*}$ \\
\hline & & 12 & 1.19 & 0.34 & 0.80 & 0.28 & $\begin{array}{l}-0.39 \\
\end{array}$ & 0.35 & $0.006 *$ \\
\hline & & 13 & 1.13 & 0.24 & 0.72 & 0.12 & -0.41 & 0.20 & $\leq 0.001^{*}$ \\
\hline & & 21 & 1.59 & 0.40 & 0.77 & 0.21 & -0.82 & 0.46 & $\leq 0.001^{*}$ \\
\hline & & 22 & 1.22 & 0.20 & 0.82 & 0.27 & -0.40 & 0.21 & $\leq 0.001^{*}$ \\
\hline & & 23 & 1.25 & 0.17 & 0.79 & 0.18 & -0.46 & 0.22 & $\leq 0.001^{*}$ \\
\hline & & 31 & 1.12 & 0.27 & 0.69 & 0.16 & -0.43 & 0.36 & $0.004 *$ \\
\hline & & 32 & 1.30 & 0.24 & 0.74 & 0.24 & -0.56 & 0.30 & $\leq 0.001 *$ \\
\hline & & 33 & 1.39 & 0.50 & 0.85 & 0.31 & -0.54 & 0.52 & $0.009 *$ \\
\hline & & 41 & 1.09 & 0.34 & 0.73 & 0.20 & -0.35 & 0.41 & $0.024 *$ \\
\hline & & 42 & 1.30 & 0.26 & 0.74 & 0.25 & -0.55 & 0.36 & $0.001 *$ \\
\hline & & 43 & 1.30 & 0.17 & 0.82 & 0.31 & -0.49 & 0.26 & $\leq 0.001 *$ \\
\hline & \multirow[t]{12}{*}{ Middle } & 11 & 2.35 & 0.73 & 1.77 & 0.89 & -0.58 & 0.51 & $0.006^{*}$ \\
\hline & & 12 & 1.82 & 0.50 & 1.23 & 0.50 & -0.59 & 0.75 & $0.034 *$ \\
\hline & & 13 & 2.25 & 0.61 & 1.45 & 0.50 & -0.80 & 0.65 & $0.004 *$ \\
\hline & & 21 & 2.21 & 1.05 & 1.80 & 1.05 & -0.41 & 0.48 & $0.023^{*}$ \\
\hline & & 22 & 1.97 & 0.56 & 1.40 & 0.55 & -0.57 & 0.54 & $0.009 *$ \\
\hline & & 23 & 2.31 & 0.34 & 1.66 & 0.66 & -0.65 & 0.65 & $0.011^{*}$ \\
\hline & & 31 & 1.24 & 0.48 & 0.82 & 0.17 & -0.42 & 0.54 & $0.037 *$ \\
\hline & & 32 & 1.31 & 0.39 & 0.91 & 0.27 & -0.40 & 0.35 & $0.005 *$ \\
\hline & & 33 & 1.77 & 0.63 & 1.10 & 0.26 & $\begin{array}{l}-0.68 \\
\end{array}$ & 0.63 & $0.008^{*}$ \\
\hline & & 41 & 1.27 & 0.36 & 0.87 & 0.25 & -0.40 & 0.41 & $0.012 *$ \\
\hline & & 42 & 1.39 & 0.37 & 0.98 & 0.26 & -0.41 & 0.37 & $0.007^{*}$ \\
\hline & & 43 & 1.75 & 0.46 & 1.28 & 0.40 & -0.47 & 0.27 & $\leq 0.001^{*}$ \\
\hline & \multirow[t]{12}{*}{ Apical } & 11 & 3.36 & 0.83 & 4.10 & 1.73 & 0.74 & 1.51 & $0.155 \mathrm{NS}$ \\
\hline & & 12 & 2.58 & 0.75 & 2.46 & 0.92 & -0.12 & 0.91 & $0.698 \mathrm{NS}$ \\
\hline & & 13 & 3.30 & 0.83 & 3.20 & 0.80 & -0.10 & 0.54 & $0.586 \mathrm{NS}$ \\
\hline & & 21 & 3.11 & 1.15 & 3.79 & 1.60 & 0.68 & 1.20 & $0.107 \mathrm{NS}$ \\
\hline & & 22 & 2.71 & 0.61 & 2.51 & 0.89 & -0.20 & 0.69 & $0.381 \mathrm{NS}$ \\
\hline & & 23 & 3.54 & 0.40 & 3.08 & 0.97 & -0.46 & 0.75 & $0.084 \mathrm{NS}$ \\
\hline & & 31 & 1.80 & 0.51 & 1.68 & 0.95 & -0.11 & 0.56 & $0.540 \mathrm{NS}$ \\
\hline & & 32 & 1.78 & 0.46 & 1.58 & 0.69 & -0.20 & 0.45 & $0.186 \mathrm{NS}$ \\
\hline & & 33 & 1.91 & 0.83 & 1.72 & 0.80 & -0.19 & 0.81 & $0.487 \mathrm{NS}$ \\
\hline & & 41 & 1.74 & 0.85 & 1.72 & 0.98 & -0.01 & 0.52 & $0.938 \mathrm{NS}$ \\
\hline & & 42 & 1.81 & 0.73 & 1.59 & 1.00 & -0.22 & 0.69 & $0.342 \mathrm{NS}$ \\
\hline & & 43 & 1.90 & 0.80 & 1.80 & 0.87 & -0.09 & 0.45 & $0.538 \mathrm{NS}$ \\
\hline
\end{tabular}

*= Significant, NS=Non-significant.

The labial bone thickness at the cervical third of the upper canines did not show a significant change which agreed with the finding of Ahn et al., ${ }^{(7)}$ and this may be attributed to the prominence of the canine root which influenced the overlying bone thickness as bone had a tendency to be thinner where the roots were prominent. ${ }^{(15)}$
The results of the cervical labial bone thickness of the upper anteriors in group II corresponded with previous studies ${ }^{(6,10)}$ which demonstrated no significant difference in the cervical labial bone thickness after retraction. For the lower anteriors, both groups showed a significant decrease in the cervical labial bone thickness after retraction which agreed with previous studies. ${ }^{6,10)}$ 
Concerning the labial bone thickness in the middle third, both groups revealed no significant difference in bone thickness after retraction except the upper left lateral incisor which demonstrated a significant decrease in group I. This finding suggested that bone resorption in the outer labial cortical plate was faster than bone apposition in the inner labial cortical plate which might be caused by increased RAP response in group I more than group II as reported in another study. ${ }^{(16)}$ This observation was detected in the lateral incisor as it demonstrated less periodontal ligament support area than those of the central incisor and canine, therefore, the tooth would receive more forces than the other teeth because the forces were distributed equally during retraction. This finding was not noticed in the lower incisors because of the similarity of the periodontal ligament area. These findings were consistent with previous studies ${ }^{(6,10)}$ which reported decreased middle labial bone thickness of upper lateral incisors after retraction. In contrast to the current results, other studies ${ }^{(7,11)}$ reported a significant increase in the labial bone thickness at the middle third that might be attributed to increased tension in those areas. Regarding the labial bone thickness in the apical third, both groups showed no significant change after retraction which was in agreement with other studies. ${ }^{(6-8,10)}$

The results of the current study showed a significant decrease in the lingual bone thickness at the cervical and middle thirds of all anteriors after en-masse retraction in both groups. This finding is consistent with other studies ${ }^{(5-7,9-12)}$ which reported reduced alveolar bone thickness in the direction of tooth movement. Bone remodeling is slow and might take years to regenerate as documented in a recent case report. ${ }^{(12)}$
Considering the apical bone thickness on the lingual side, group I demonstrated no significant change except the upper and lower canines which showed a significant decrease. Group II showed no significant change after retraction. Sarikaya et al., ${ }^{(6)}$ and Yodthong et al., ${ }^{(8)}$ reported similar results. The bone thickness at the apical region of the canines was significantly decreased in group I which indicated more bone resorption than the incisors as the root of the canine is longer and more prominent. Moreover, bone resorption might be increased by a more profound RAP response induced by the flap technique. ${ }^{(16)}$ This finding is consistent with the results of Ahn et al., ${ }^{(7)}$ and Yinghong et al., ${ }^{(11)}$ who reported decreased bone thickness in all lingual areas after retraction.

\section{CONCLUSION}

1- Concerning the cervical labial thickness changes, the upper incisors showed a significant increase after en-masse retraction in the corticotomy group while revealed no significant change in the piezocision group. The lower anteriors showed a significant decrease in both groups.

2- The lingual bone thickness of the upper and lower anterior teeth demonstrated a significant decrease in the cervical and middle thirds after retraction in both groups.

\section{REFERENCES}

1. Baek SH, Kim BH. Determinants of successful treatment of bimaxillary protrusion: orthodontic treatment versus anterior segmental osteotomy. J Craniofac Surg. 2005;16:234-46.

2. Mimura H. Treatment of severe bimaxillary protrusion with miniscrew anchorage: treatment and complication. Aust Ortho J. 2008;24:156-63. 
3. Nahm KY, Kang JH, Moon SC, Choi YS, Kook YA, Kim SH, Huang JC. Alveolar bone loss around incisors in Class 1 bidentoalveolar protrusion patients: a retrospective three-dimensional cone beam CT study. Dentomaxillofac Radiol. 2012;41:481-8.

4. Bimstein E, Crevoisier RA, King DL. Changes in the morphology of the buccal alveolar bone of protruded permanent mandibular incisors secondary to orthodontic alignment. Am J Orthod Dentofacial Orthop.1990;97:427-30.

5. Vardimon AD, Oren E, Ben-Bassat Y. Cortical bone remodeling/tooth movement ratio during maxillary incisor retraction with tip versus torque movements. Am J Orthod Dentofacial Orthop. 1998;114:520-9.

6. Sarikaya S, Haydar B, Ciger S, Ariyurek M. Changes in alveolar bone thickness due to retraction of anterior teeth. Am J Orthod Dentofacial Orthop. 2002; 122: 15-26.

7. Ahn HW, Moon SC, Baek SH. Morphometric evaluation of changes in the alveolar bone and roots of the maxillary anterior teeth before and after en masse retraction using cone-beam computed tomography. Angle Orthod. 2013;83: 212-21.

8. Yodthong N, Charoemratrote C, Leethanakul C. Factors related to alveolar bone thickness during upper incisor retraction. Angle Orthod. 2013;83:394-401.

9. Picanço PR, Valarelli FP, Cançado RH, Freitas KM, Picanço GV. Comparison of the changes of alveolar bone thickness in maxillary incisor area in extraction and non-extraction cases: Computerized tomography evaluation. Dental Press J Orthod. 2013;18:91-8.
10. Nayak Krishna U S, Shetty A, Girija M P, Nayak R. Changes in alveolar bone thickness due to retraction of anterior teeth during orthodontic treatment: A cephalometric and computed tomography comparative study. Indian J Dent Res. 2013;24:736-41.

11. Yinghong L, Zeyuan Z, Kui Z, Caomin T, Jun W. Morphometric evaluation of changes in the alveolar bone of adolescents with bimaxillary protrusion via cone beam computed tomography. West China journal of stomatology. 2016;34:78-84

12. Bae SM, Kim HJ, Kyung HM. Long-term changes of the anterior palatal alveolar bone after treatment with bialveolar protrusion, evaluated with computed tomography. Am J Orthod DentofacialOrthop.2018;153:108-17.

13. Lee JK, Chung KR, Baek SH. Treatment outcomes of orthodontic treatment, corticotomy-assisted orthodontic treatment, and anterior segmental osteotomy for bimaxillary dentoalveolar protrusion. Plast Reconstr Surg. 2007; 120:1027-36.

14. De Angelis V. Observations on the response of alveolar bone to orthodontic force. Am J Orthod. 1970;58:284-94.

15. Leung CC, Palomo L, Griffith R, Hans MG. Accuracy and reliability of cone-beam computed tomography for measuring alveolar bone height and detecting bony dehiscences and fenestrations. Am J Orthod Dentofacial Orthop. 2010;137:S109-19.

16. Abbas NH, Sabet NE, Hassan IT. Evaluation of corticotomy-facilitated orthodontics and piezocision in rapid canine retraction. Am J Orthod Dentofacial Orthop. 2016;149:473-80. 